\title{
CT in COPD: just a pretty picture or really worth a thousand words (or dollars)?
}

\author{
Don D Sin, ${ }^{1,2}$ Jonathon Leipsic, ${ }^{3}$ S F Paul Man ${ }^{1,2}$
}

We like pretty pictures and, in pulmonary medicine, we use computed axial tomography (CT) to generate pretty pictures to help us diagnose and manage patients with respiratory complaints. In 2007 more than 10 million chest CT scans were performed across the USA, representing an astounding $11000 \%$ increase in the CT rate since $1980 .{ }^{1}$ CT scans rely on ionising radiation to generate images, and recent estimates suggest that CT scans may be responsible for $24 \%$ of the total 'background' radiation to which the population is exposed in a given year. ${ }^{2}$ Thankfully, there are several large-scale efforts to reduce the radiation exposure related to CT scans and to mitigate the health risks imposed by ionising radiation. ${ }^{3}$ What is not being adequately addressed is the issue of economic costs (and benefits) of CT scans. CT scans are expensive for patients and to the healthcare system, with prices ranging from $\$ 500$ to $\$ 1500$ per scan. ${ }^{4}$ Recently, the cost-effectiveness ratio of lung cancer screening with CT scans was reported to be $\$ 2.3$ million dollars per quality-adjusted life saved, ${ }^{5}$ providing us with a sobering reminder that these 'pretty pictures' are not without significant costs.

Chronic obstructive pulmonary disease (COPD) is a condition that lends itself to anatomical medical imaging. For practical reasons, COPD is largely defined based on spirometric criteria. However, there is general discontent with this approach because spirometric measurements are relatively insensitive and correlate only very loosely with histological abnormalities or with patient symptoms or outcomes. $^{6} \quad$ Furthermore, spirometric measurements are poorly responsive to

\footnotetext{
${ }^{1}$ UBC James Hogg Research Center, St Paul's Hospital, University of British Columbia, Vancouver, British Columbia, Canada; ${ }^{2}$ Department of Medicine, Respiratory Division, University of British Columbia, Vancouver, British Columbia, Canada; ${ }^{3}$ Department of Radiology, University of British Columbia, Vancouver, British Columbia, Canada

Correspondence to Don D Sin, St Paul's Hospital, University of British Columbia, Room 8442, 1081 Burrard Street, Vancouver, BC V6Z 1Y6, Canada; don.sin@hli.ubc.ca
}

medical interventions (even those that are known to improve morbidity and mortality) and cannot discriminate the major pathological subphenotypes of COPD-emphysema and small airways disease. $^{6}$ More complete and elaborate lung function measurements provide more information but they are expensive, timeconsuming, difficult to standardise and relatively inaccessible beyond large hospitals, making them non-user friendly for many practising physicians. On the other hand, high-resolution CT (HRCT) provides the clinicians with excellent anatomical detail and takes away the veil and mystery of lung function measurements. With continued evolution and refinement of this technology, the hope is that HRCT will one day complement (or even replace) lung function measurements in diagnosing and managing patients with COPD in routine clinical practice. However, is this notion realistic?

It is now well established that some current and former smokers with no or minimal pulmonary symptoms can have normal forced expiratory volume in $1 \mathrm{~s}$ $\left(F_{1}\right)$ and ratio of $\mathrm{FEV}_{1}$ to forced vital capacity (FVC) but still harbour significant emphysematous changes in their lungs. ${ }^{7}$ The clinical relevance of this observation, however, was unknown. The study by Mohamed Hoesein et al offers an answer to this clinical conundrum. Using data from 2085 current and former heavy smokers who enrolled in the Dutch-Belgian Lung Cancer Screening Trial (NELSON), Mohamed Hoesein and colleagues showed that individuals with the largest burden of CT-based 'emphysema' experienced the fastest decline in lung function over 3 years of follow-up. This effect was independent of age, smoking status or baseline lung function of these individuals. ${ }^{8}$ These data are in keeping with those of Yuan et al who showed that lung 'overinflation' was associated with a rapid decline in $\mathrm{FEV}_{1}$. However, owing to the small sample study ( $\mathrm{n}=143)$, Yuan et al could not show a relationship with more traditional CTbased measures of emphysema. ${ }^{7}$ The physiological rationale for the relationship between CT-based emphysema, lung 'overinflation' and rapid decline in lung function is obscure, but several possibilities exist. Although, in general, mild degrees of lung emphysema do not result in airflow limitation, they can lead to air trapping and lung 'overinflation'.' However, with emphysema progression, airflow limitation ensues owing to reductions in elastic recoil pressure and loss of alveolar attachments ${ }^{10}$ which leads to narrowing and premature closure of airways. Alternatively, it is possible that CT-based measures of emphysema may just be a marker of pathological changes in the small airways (eg, remodelling and fibrosis) that may be the more salient drivers of COPD progression but cannot be well visualised on HRCT scans.

The study by Mohamed Hoesein et al (see page 782) has several limitations that deserve emphasis. First, emphysema is a pathological (and not a radiological) diagnosis and in this study there was no histological confirmation of CT-based assessment of emphysema. Furthermore, the study used only one metric to evaluate emphysema-the extent of low attenuation areas on the CT scan. While this is commonly used, inclusion of other salient radiographic features of emphysema such as low attenuation cluster analysis, the presence of gas trapping and the regional distribution of the low attenuation areas across the lobes would have enhanced the accuracy of the definition. ${ }^{11}$ Second, only men were studied so these data cannot be generalised to the female COPD population. Third, only one follow-up spirometric value was obtained, making it possible that 'regression to the mean' could have confounded the results.

The NELSON trial is a lung cancer screening study which uses low-dose CT scans for early detection of malignant tumours. The final results from this trial are not expected until 2015. ${ }^{12}$ Thus, the current study by Mohamed Hoesein et al could not provide any data on the relationship between CT measures of emphysema and the subsequent risk of lung cancer, which is the leading cause of mortality in patients with mild COPD. ${ }^{13}$ However, a previous study by Wilson et al ${ }^{14}$ which used CT scans collected in a different lung cancer screening programme suggests that smokers with emphysema on CT scans have a significantly increased risk of lung cancer, independent of their lung function. Together, the data by Mohamed Hoesein et al and Wilson et al indicate that CT-based measurements of emphysema in smokers 
with normal or near normal lung function are not just pretty pictures but are clinically important because they identify individuals at high risk of COPD progression and lung cancer. In such individuals it may be highly cost-effective to intervene with aggressive tobacco treatment programmes and with close observation and follow-up.

On 4 November 2010 the US National Cancer Institute (NCI) released the initial results from the National Lung Screening Trial (NSLT), showing a reduction of $20 \%$ in lung cancer mortality and a reduction of $7 \%$ in total mortality among exsmokers and current smokers screened with low-dose CT compared with those screened with chest x-rays. ${ }^{15}$ Notwithstanding the costs associated with HRCT scans, these and other data on screening CT for lung cancer will probably lead to an exponential increase in the number of thoracic HRCT scans that will be performed over the next few years. This will present new opportunities for clinical care and research for the respiratory community. In addition to using these CT scans as tools for lung cancer screening, the data by Mohamed Hoesein et al suggest that chest physicians can also use them to identify high-risk patients who are likely to experience rapid COPD progression and to aggressively treat them for tobacco addiction (if they are current smokers) and to institute therapies for their COPD when clinically appropriate. With agreed protocols to acquire and analyse the images, the widespread use of thoracic CT scans may also provide a tremendous opportunity for researchers to understand the natural history of COPD in individuals with 'subclinical' COPD (based on CT only) and its associated comorbidities such as lung cancer, cardiovascular disease and osteoporosis. Perhaps, by doing so, we can maximise the value of screening lung CT scans and make these pretty pictures worth a thousand words (or dollars)!

Acknowledgements DDS is the holder of a Canada Research Chair in COPD and a senior scholar with the Michael Smith Foundation for Health Research.

\section{Competing interests None.}

Patient consent Obtained.

Contributors All of the authors were involved in the drafting and revising of the editorial.

Provenance and peer review Commissioned; externally peer reviewed.

Published Online First 14 May 2011

Thorax 2011;66:741-742

doi:10.1136/thx.2011.161430

\section{REFERENCES}

1. Smith-Bindman R, Lipson J, Marcus R, et al. Radiation dose associated with common computed tomography examinations and the associated lifetime attributable risk of cancer. Arch Intern Med 2009;169:2078-86.

2. Schenkman L. Radiology. Second thoughts about CT imaging. Science 2011;331:1002-4.

3. Leipsic J, Nguyen G, Brown J, et al. A prospective evaluation of dose reduction and image quality in chest CT using adaptive statistical iterative reconstruction. AJR Am J Roentgenol 2010;195:1095-9.

4. Canada Diagnostic Centers. Fee schedule 2011. http://www.canadadiagnostic.com/fee-schedulescans.php (accessed 11 Apr 2011).
5. Weinstein MC, Skinner JA. Comparative effectiveness and health care spending -implications for reform. N Engl J Med 2010;362:460-5.

6. Cazzola M, MacNee W, Martinez FJ, et al. Outcomes for COPD pharmacological trials: from lung function to biomarkers. Eur Respir $\mathrm{J}$

2008;31:416-69

7. Yuan R, Hogg JC, Pare PD, et al. Prediction of the rate of decline in $\mathrm{FEV}(1)$ in smokers using quantitative computed tomography. Thorax 2009:64:944-9.

8. Mohamed Hoesein FA, de Hoop B, Zanen P, et al. CT-quantified emphysema in male heavy smokers: association with lung function decline. Thorax 2011;66:782-7.

9. Petty TL, Silvers GW, Stanford RE. Mild emphysema is associated with reduced elastic recoil and increased lung size but not with air-flow limitation. Am Rev Respir Dis 1987;136: 867-71.

10. Petty TL, Silvers GW, Stanford RE. Radial traction and small airways disease in excised human lungs. Am Rev Respir Dis 1986;133:132-5.

11. Gietema HA, Muller NL, Nasute Fauerbach PV, et al. Quantifying the extent of emphysema: factors associated with radiologists' estimations and quantitative indices of emphysema severity using the ECLIPSE cohort. Acad Radiol 2011;18: $661-71$.

12. van Klaveren RJ, Oudkerk M, Prokop $M$, et al. Management of lung nodules detected by volume CT scanning. N Engl J Med 2009;361:2221-9.

13. Anthonisen NR, Connett JE, Kiley JP, et al. Effects of smoking intervention and the use of an inhaled anticholinergic bronchodilator on the rate of decline of FEV1. The Lung Health Study. JAMA 1994:272:1497-505

14. Wilson Do, Weissfeld JL, Balkan A, et al. Association of radiographic emphysema and airflow obstruction with lung cancer. Am J Respir Crit Care Med 2008;178:738-44.

15. National Cancer Institute. National Lung Cancer Screening Trial (NLST) initial results: fast facts. 2010. http://www.cancer.gov/newscenter/ pressreleases/2011/NLSTFastFacts laccessed 9 May 2011).

tion levels are high by global standards BOS is the major risk factor for death after lung transplantation, so it is not surprising that exposure to air traffic pollution, defined by residential proximity to a major road, was also a risk factor for death after transplantation. Importantly, other potential risk factors were rigorously examined to prevent confounding and the relationship was highly significant regardless of whether distance categories from a main road were expressed as a dichotomous or continuous variable. In addition, there was a strong relationship between distance from a main road and the finding of a bronchoalveolar lavage neutrophilia, an association that implies but does not prove an aetiological link. Importantly, it makes biological sense. The findings are illuminating and may explain in part some of the reported
Correspondence to Professor Allan R Glanville, The Lung Transplant Unit, Xavier 4, St Vincent's Hospital, Victoria Street, Darlinghurst, NSW 2010, Australia; aglanville@stvincents.com.au development of the bronchiolitis oblit erans syndrome (BOS) in a large and wellcharacterised sample of lung transplant recipients from a region where air pollu- 